\title{
ANNIHILATOR CHARACTERIZATIONS OF DISTRIBUTIVITY, MODULARITY AND SEMIMODULARITY
}

\author{
By \\ Juhani Nieminen and Matti Peltola
}

The concept of geodetic annihilators was introduced in [3]. This concept is based on the graph theoretic properties of the Hasse diagram of a finite lattice. The result of [3, Thm. 3] shows that every geodetic annihilator in a finite semimodular lattice is an intersection of prime geodetic annihilators. This and appropriate additional conditions of geodetic annihilators characterize semimodularity, modularity and distributivity in finite lattices. The graphs of these lattices are also characterized.

\section{Characterizations of finite lattices}

Mandelker introduced in [2] the concept of an annihilator in lattices: an annihilator $\langle a, b\rangle$ of $a$ with respect to $b$ is the set $\{x \mid x \wedge a \leq b\}$. The dual annihilator $\langle a, b\rangle_{d}$ is the set $\{y \mid y \vee a \geq b\}$. We shall consider in this paper finite lattices only.

Let $L$ be a lattice. We denote the undirected Hasse diagram graph of a lattice $L$ by $G_{L}$ and call it briefly the graph of the lattice $L$. The distance $d(a, b)$ between two elements (vertices) $a$ and $b$ in a graph is the length of the shortest $a-b$ path. In graph theory, a shortest path is frequently called a geodesic. We call a set $\langle a, b\rangle_{g}$ of a lattice $L, a, b \in L$, a geodetic annihilator, briefly a $g$-annihilator, if $\langle a, b\rangle_{g}=\left\{x \mid b\right.$ is on an $x-a$ geodesic in $\left.G_{L}\right\}$. A set $B \subset L$ is order convex, if for any two elements $b, c \in B$ with $b \leq c$ every element $x$ satisfying the relation $b \leq$ $x \leq c$ belongs to $B$. A set $B$ of vertices in a graph is distance convex, if for any two vertices $b, c \in B$ every vertex on any $b-c$ geodesic belongs to $B$. We first briefly recall some results proved in [3], which are necessary for obtaining results of this note.

Received August 28, 1997.

Revised July 1, 1998. 
LeMma 1 ([3, Thm. 1]). In a finite distributive lattice $L$ the equality $\langle a, b\rangle_{g}=\langle a, b\rangle \cap\langle a, b\rangle_{d}$ holds for every pair $a, b \in L$.

Mandelker proved that a lattice is distributive if and only if the annihilator $\langle a, b\rangle$ is an ideal for any two elements $a$ and $b$ [2, Thm. 1]. The dual of this result holds also, and so every $g$-annihilator in a distributive lattice is, as the intersection of an ideal and a dual ideal, an order convex sublattice. As known, an order convex sublattice of a distributive lattice is also distance convex.

As usual, we say that an element $a$ covers an element $b$, in symbols $a \succ b$, if $a>b$ and if $a \geq c \geq b$ implies $a=c$ or $b=c$. A $g$-annihilator $\langle a, b\rangle_{g}$ is called prime, if $\langle a, b\rangle_{g}=L$ or

$$
\langle a, b\rangle_{g} \cap\langle b, a\rangle_{g}=\varnothing \quad \text { and } \quad\langle a, b\rangle_{g} \cup\langle b, a\rangle_{g}=L .
$$

As one can easily show, (see e.g. the proof of Theorem 2 in [3]), the relation $\langle a, b\rangle_{g} \cap\langle b, a\rangle_{g}=\varnothing$ holds in every graph (and thus in every lattice, too) when $a \neq b$. Thus the condition $\langle a, b\rangle_{g} \cap\langle b, a\rangle_{g}=\varnothing$ can be replaced by the condition $a \neq b$.

A $g$-annihilator $\langle a, b\rangle_{g}$ with $a \neq b$ is prime only if $a \succ b$ or $b \succ a$. Indeed, if neither $a \succ b$ nor $b \succ a$ holds, there is an $a-b$ geodesic containing an element $x \neq a, b$, and by the definition of a $g$-annihilator, $x$ belongs neither to $\langle a, b\rangle_{g}$ nor to $\langle b, a\rangle_{g}$. Then $\langle a, b\rangle_{g} \cup\langle b, a\rangle_{g} \neq L$, and thus $\langle a, b\rangle_{g}$ cannot be prime. It has been proved in [3, Thm. 3]: "In a finite lattice $L$ satisfying the Jordan-Hölder condition, every $g$-annihilator is an intersection of prime $g$-annihilators". This result implies the following lemma.

LEMMA 2. In a finite semimodular lattice $L$, every $g$-annihilator $\langle a, b\rangle_{g}$ is an intersection of prime g-annihilators.

Now we can prove the first characterization.

THEOREM 3. A finite lattice $L$ is semimodular if and only if the following conditions hold

(i) every $g$-annihilator in $L$ is an intersection of prime g-annihilators;

(ii) if $b \prec a$, then $b \vee x \in\langle a, b\rangle_{g}$ for every $x \in\langle a, b\rangle_{g}$.

Proof. Assume first that $L$ is semimodular. The property (i) follows from Lemma 2, and so it remains to prove (ii). Let $b \prec a$ and $x \in\langle a, b\rangle_{g}$. We prove the assertion by induction on the distance $d(x, b)$. If $d(x, b)=0$ or 1 , then either $x=x \vee b$ or $b=x \vee b$, whence an $x-b$ geodesic goes over $x \vee b$, and thus $x \vee b \in\langle a, b\rangle_{g}$. Assume now that one $y-b$ geodesic goes over $y \vee b$ for all 
elements $y \in\langle a, b\rangle_{g}$ with $d(y, b)<n$, and let $d(x, b)=n$. Let $x, y_{1}, y_{2}, \ldots$, $y_{n-1}, b, a$ be an $x-a$ geodesic, where, let us say, $y_{k}=y_{1} \vee b$ and $y_{1} \prec y_{2} \prec, \ldots, \prec y_{k}=y_{1} \vee b$. If $x \prec y_{1}$, then $x \vee b=y_{1} \vee b$, and $x \vee b \in$ $\langle a, b\rangle_{g}$. If $x \succ y_{1}$, then $x \vee y_{2} \succ x, y_{2}$, and by the same reason we obtain: $x \prec x \vee y_{2} \prec x \vee y_{3} \prec \cdots \prec x \vee y_{k} \succ y_{k}$. Now $x \vee y_{k}=x \vee y_{1} \vee b=x \vee b$, and the $x-y_{1}-y_{2}-\cdots-y_{k}$ geodesic has the same length as the $x-x \vee y_{2}-$ $x \vee y_{3}-\cdots-x \vee y_{k}-y_{k}$ geodesic. Hence $x \vee b \in\langle a, b\rangle_{g}$, and (ii) follows.

Assume conversely that the properties (i) and (ii) hold in $L$. We shall prove the semimodularity of $L$ by induction on the length of the longest $0-1$ chain in $L$. If the longest $0-1$ chain is of length one, then $L$ is clearly semimodular. Assume that $L$ is semimodular if the lenght of the longest $0-1$ chain is less than $n$, and let $L$ be a lattice where the length of this longest chain is $n$. Assume that $e, f \succ e \wedge f$. The intersection property of (i) goes down to the order convex sublattices of $L$ as well as the property (ii). Hence, if $e \wedge f \neq 0$ or $e \vee f \neq 1$, the length of the longest $0-1$ chain in $[e \wedge f]$ (or in $[e \vee f]$ ) is less than $n$, and the relation $e \vee f \succ e, f$ follows from the induction assumption. Thus we assume that $0=e \wedge f \prec e, f$ and $e \vee f=1$. We may also assume that an $x-y$ geodesic with $x$ from $0-e-1$ chain and $y$ from $0-f-1$ chain always goes either over the element 1 or the element 0 . Choose now two elements $a_{0}$ and $b_{0}$ as follows: $a_{0} \succ b_{0}=e$. If $a_{0}=1$, choose $a_{0} \succ b_{0}=f$, and if also in this case $a_{0}=1$, the relation $e, f \prec e \vee f$ follows. So we may assume that $1>a_{0} \succ b_{0}=e$. Now clearly $f \in\left\langle a_{0}, b_{0}\right\rangle_{g}$, and by (ii), $f \vee b_{0}=1 \in\left\langle a_{0}, b_{0}\right\rangle_{g}$. If there is not a $1-a_{0}$ geodesic over $b_{0}$, we have a contradiction, and if there is a $1-a_{0}$ geodesic over $b_{0}$, choose new elements $a_{1}$ and $b_{1}$ as follows: $a_{1} \succ b_{1}=a_{0} \succ b_{0}=e \succ 0$. By continuing the choosing process, we certainly obtain a contradiction (at least in the case $\left.1=a_{j} \succ b_{j}=a_{j-1} \succ b_{j-1} \succ b_{j-2} \succ \cdots \succ b_{0}=e\right)$, where $1 \in\left\langle a_{j}, b_{j}\right\rangle_{g}$ by the condition (ii) and $1 \in\left\langle b_{j}, a_{j}\right\rangle_{g}$ by the definition of a $g$-annihilator. Thus this case is absurd, and the semimodularity of $L$ follows.

By dualizing the condition (ii), a characterization of the dual semimodularity is obtained. A finite lattice is modular if and only if it is semimodular and dually semimodular. Thus Theorem 3 and its dual imply the next characterization.

THEOREM 4. A finite lattice $L$ is modular if and only if the following conditions hold

(i) every g-annihilator in $L$ is an intersection of prime g-annihilators;

(ii) if $b \prec a$ then $b \vee x, b \wedge x \in\langle a, b\rangle_{g}$ for every $x \in\langle a, b\rangle_{g}$.

Finally we characterize the distributivity. 
THEOREM 5. A finite lattice $L$ is distributive if and only if the following conditions hold

(i) every g-annihilator in $L$ is an intersection of prime g-annihilators;

(ii) every g-annihilator $\langle a, b\rangle_{g}$ is an order convex sublattice of $L$

Proof. Let $L$ be distributive. The property (i) follows from Lemma 2, and Lemma 1 and the considerations immediately after it prove the validity of (ii).

Assume conversely that (i) and (ii) hold for $L$. By Theorem 4, $L$ is at least modular, and as one can prove, if a finite lattice is modular and nondistributive, it contains the least modular and nondistributive lattice, the diamond $M_{3}$, as a sublattice. Let $M_{3}=\{a, b, c, d, e\}$ with three noncomparable elements $b, c$ and $d$ and with the order relations: $a<b<e, a<c<e$ and $a<d<e$. Because of the distance properties of a finite modular lattice, $\langle b, e\rangle_{g} \supset\{e, c, d\}$, and $a=c \wedge d \notin$ $\langle b, e\rangle_{g}$, which contradicts (ii). Thus $L$ cannot contain $M_{3}$ as a sublattice, and the distributivity of $L$ follows.

As the proof above shows, the condition can be reduced to the form "every $g$-annihilator $\langle a, b\rangle_{g}$ is a sublattice of $L$ ".

\section{The graphs of finite lattices}

The definitions of a $g$-annihilator and a prime $g$-annihilator do not depend on lattice operations, and so we can speak about $g$-annihilators and prime $g$ annihilators in finite undirected graphs, too.

A median $m$ for vertices $x, y$ and $z$ is a vertex satisfying the equations $d(x, y)=d(x, m)+d(m, y), d(x, z)=d(x, m)+d(m, z)$ and $d(y, z)=d(y, m)+$ $d(m, z)$. As well known [1], the median of any three elements $x, y$ and $z$ exists, is unique and is equal to $(x \wedge y) \vee(x \wedge z) \vee(y \wedge z)$ in a finite distributive lattice. In a finite modular lattice a median exists for all three elements but it need not be unique. In a dually semimodular finite lattice there are triples having no median but the median $m(x, y, 0)$ of $x, y$ and 0 exists for all $x$ and $y$, it is unique and equal to $x \wedge y$. Dually, in a finite semimodular lattice the unique median $m(x, y, 1)$ exists for all pairs $x$ and $y$ and is equal to $x \vee y$. Now we can present our first characterization for graphs.

THEOREM 6. A finite undirected graph $G$ is isomorphic to the graph $G_{L}$ of a dually semimodular finite lattice $L$ if and only if the following conditions hold

(i) every g-annihilator in $G$ is an intersection of prime g-annihilators;

(ii) there are two vertices $p$ and $q$ in $G$ such that for no vertex $x \neq p, q$ the vertex $p$ lies on an $x-q$ geodesic and the vertex $q$ lies on an $x-p$ geodesic; 
(iii) a unique median $m(x, y, p)$ exists for all pairs $x$ and $y$ of vertices in $G$, and if $x, y \in\langle p, z\rangle_{g}$, then also $(x, y, p) \in\langle p, z\rangle_{g}$;

(iv) if $a$ and $b$ are adjacent and $b \in\langle p, a\rangle_{g}$, then the median $m(x, b, p)$ belongs to $\langle a, b\rangle_{g}$ for all $x \in\langle a, b\rangle_{g}$.

Proof. Assume that $G$ satisfies the properties (i)-(iv). We order the vertices of $G$ as follows: $x \leq y \Leftrightarrow\langle p, x\rangle_{g} \supset\langle p, y\rangle_{g}$. According to the set theoretical inclusion, the order defined above is a partial order with $p$ as the least element. The definition implies that $x \leq y \Leftrightarrow y \in\langle p, x\rangle_{g}$. Assume that two vertices $x$ and $y$ of $G$ have a common lower bound $b$, which implies that $x, y \in\langle p, b\rangle_{g}$. Now (iii) implies that $m(x, y, p) \in\langle p, b\rangle_{g}$, and thus $b \leq m(x, y, p)$. Clearly, $m(x, y, p) \leq x, y$. Because $G$ is finite, the considerations above imply that $m(x, y, p)$ is the unique maximal lower bound for $x$ and $y$, and so the order defined above is a meetsemilattice order. The condition (ii) and the definition of the order relation imply that $x \leq q$ for all vertices $x$ of $G$, whence $q$ is the greatest element, and so the vertices of $G$ constitute a lattice $L$. The condition (iv) says that if $a$ covers $b$, then $x \wedge b \in\langle a, b\rangle_{g}$ for all $x \in\langle a, b\rangle_{g}$. This, (i) and the dual of Theorem 3 imply that $L$ is dually semimodular.

If $x$ and $y$ are adjacent in $G$, then there is a median $m(x, y, p)$ which lies on an $x-y$ geodesic, and thus either $x=m(x, y, p)=x \wedge y$ or $y=m(x, y, p)=$ $x \wedge y$. Assume that $x>y=x \wedge y$. The element $x$ covers $y$, because otherwise there is a vertex $z$ such that one $x-y-p$ geodesic is also and $x-z-y-p$ geodesic, which is absurd when $x$ and $y$ are adjacent. So we can conclude that $x$ covers $y$ or $y$ covers $x$ if and only if $x$ and $y$ are adjacent in $G$. Therefore the graph $G$ and the graph of the lattice $L$ are isomorphic, and the first part of the theorem follows.

The converse proof is obtained easily by putting $p=0, q=1$, and by applying the dual of Theorem 3. This completes the proof.

Theorem 6, its dual and Theorem 4 imply the following characterization of the graphs of finite modular lattices.

THEOREM 7. A finite undirected graph is isomorphic to the graph $G_{L}$ of a finite modular lattice if and only if the following conditions hold

(i) every g-annhilator in $G$ is an intersection of prime g-annihilators;

(ii) there are two vertices $p$ and $q$ in $G$ such that for no vertex $x \neq p, q$, the vertex $p$ lies on an $x-q$ geodesic and the vertex $q$ lies on an $x-p$ geodesic;

(iii) unique medians $m(x, y, p)$ and $m(x, y, q)$ exist for all pairs $x$ and $y$ of vertices in $G$, and if $x, y \in\langle p, z\rangle_{g}$, then also $m(x, y, p) \in\langle p, z\rangle_{g}$; 
(iv) if $a$ and $b$ are adjacent and $b \in\langle p, a\rangle_{g}$, then the medians $m(x, b, p)$ and $m(x, b, q)$ belong to $\langle a, b\rangle_{g}$ for all $x \in\langle a, b\rangle_{g}$.

In a finite distributive lattice $L$, every $g$-annihilator is a distance convex set, and this property implies that $x \wedge b, x \vee b \in\langle a, b\rangle_{g}$ for all elements $x \in\langle a, b\rangle_{g}$. On the other hand, a modular lattice, where every $g$-annihilator is a distance convex set, is distributive. Hence we can substitute the condition (iv) of Theorem 7 by the condition of distance convexity and obtain the following characterization for the graphs of finite distributive lattices.

THEOREM 8. A finite undirected graph $G$ is isomorphic to the graph $G_{L}$ of a finite distributive lattice $L$ if and only if the following conditions hold

(i) every g-annihilator in $G$ is an intersection of prime g-annihilators;

(ii) there are two vertices $p$ and $q$ in $G$ such that for no vertex $x \neq p, q$, the vertex $p$ lies on an $x-q$ geodesic and the vertex $q$ lies on an $x-p$ geodesic;

(iii) unique medians $m(x, y, p)$ and $m(x, y, q)$ exist for all pairs $x$ and $y$ of vertices in $G$;

(iv) every g-annihilator $\langle a, b\rangle_{g}$ in $G$ is a distance convex set.

\section{References}

[1] Bandelt, H. J. and Hedlikova, J., Median algebras, Discrete Math. 45 (1983), 1-30.

[2] Mandelker, M., Relative annihilators in lattices, Duke Math. J. 40 (1970), 377-386.

[3] Nieminen, J., The Jordan-Hölder chain condition and annihilators in finite lattices. Tsukuba J. Math. 14 (1990), 405-411.

Department of Mathematics

Faculty of Technology

University of Oulu

PB 444

90571 Oulu

Finland 